\title{
Nonmotor Symptoms of Parkinson's Disease
}

\author{
Irena Rektorova, ${ }^{1}$ Dag Aarsland, ${ }^{2,3}$ K. Ray Chaudhuri, ${ }^{4}$ and Antonio P. Strafella ${ }^{5}$ \\ ${ }^{1}$ Movement Disorders Center, First Department of Neurology, Medical Faculty, St. Anne's University Hospital and Applied Neuroscience \\ Research Group, Central European Institute of Technology CEITEC, Masaryk University, 62500 Brno, Czech Republic \\ ${ }^{2}$ Department of Neurobiology, Care Sciences and Society, Alzheimer's Disease Research Center, Karolinska Institute, \\ 14186 Stockholm, Sweden \\ ${ }^{3}$ Centre for Age-Related Medicine, Stavanger University Hospital and Institute of Clinical Medicine, Akershus University Hospital, \\ University of Oslo, 4005 Stavanger, Norway \\ ${ }^{4}$ National Parkinson Foundation Centre of Excellence, King's College Hospital, King's College, London SE5 9RS, UK \\ ${ }^{5}$ Movement Disorder Unit and E. J. Safra Parkinson Disease Program, Toronto Western Hospital, UHN and Imaging Research Centre, \\ Centre for Addiction and Mental Health, University of Toronto, Toronto, ON, Canada M5T 2S8
}

Correspondence should be addressed to Irena Rektorova, irena.rektorova@fnusa.cz

Received 9 January 2012; Accepted 9 January 2012

Copyright (C) 2011 Irena Rektorova et al. This is an open access article distributed under the Creative Commons Attribution License, which permits unrestricted use, distribution, and reproduction in any medium, provided the original work is properly cited.

The nonmotor symptoms (NMSs) of Parkinson's disease (PD) have received a lot of attention in the last few years. Despite this fact, they have still been underrecognized and undertreated $[1,2]$. NMS may include cognitive problems, apathy, depression, anxiety, hallucinations, and psychosis as well as sleep disorders, fatigue, autonomic dysfunction, sensory problems, and pain [1]. Since these symptoms substantially contribute to patients' quality of life and are a frequent cause of hospitalization and institutionalization, the NMSs and their management have been recognized by the UK National Institute for Clinical Excellence as an important unmet need in PD [3]. Besides dopamine and Lewy-type pathology involving both striatal and extrastriatal brain regions, deficits in other neurotransmitter systems and/or other types of brain pathologies seem to play a key role in the pathogenesis of NMS in PD [4-6].

It has been clearly shown that dopaminergic treatment improves motor symptoms and the quality of life in patients with PD. However, it only partly improves some features of the NMS, and it is not free from nonmotor side effects such as hallucinations and other psychotic symptoms, irritability, sleep attacks, and impulse control disorders. Furthermore, one study found that the patients who survived at 20 years from PD diagnosis suffered mainly from the nondopaminergic symptoms including falls, choking, dysarthria, but also dementia, visual hallucinations, daytime somnolence, symptomatic postural hypotension, and urinary incontinence [7]. Therefore, the management of these nondopaminergic symptoms is a priority for research in the near future.

In addition to pharmacotherapy, high-frequency deep brain stimulation of the subthalamic nucleus (STN DBS) is a powerful surgical treatment in well-selected candidates with advanced PD. STN DBS leads to improvements in dopaminergic drug-sensitive symptoms and reductions in subsequent drug dose and dyskinesias [8]. Although quality of life improves substantially, the procedure cannot be recommended for the treatment of NMS, and it may even cause specific cognitive side effects and may increase the risk for suicide. Skilled speech and physical therapy with cueing to improve gait, cognitive therapy to improve transfers, exercises to improve balance, and training to build up muscle power and increase joint mobility are efficacious. Regular physical and mental exercise is therefore recommended for all $\mathrm{PD}$ patients [9].

As outlined above, multidisciplinary approach including both pharmacological and nonpharmacological treatment of PD is essential; however, the current delivery of allied healthcare services is inadequate, and many people who require such care are not being referred to the relevant specialist. Parkinson's Standards of Care Consensus Statement will soon be released by the European Parkinson's Disease Association and should be implemented in Europe in the near future. 
Besides symptomatic treatment, search for the diseasemodifying, neuroprotective, and restorative treatment of PD is ongoing. Despite many so far unresolved issues, geneand stem-cell-based therapies as well as immunotherapy targeting alpha-synuclein might become treatment options in the future. Development of these therapies is dependent on an accurate and comprehensive understanding of the pathogenesis and pathophysiology of PD and on the ability of very early diagnosis of premotor stages of PD. Therefore, a search for specific biomarkers (clinical, neuroimaging, biochemical, genetic) for early (premotor) diagnosis and for the disease progression is essential and large multicenter trials are underway.

This special issue is dedicated to nonmotor symptoms of PD and focuses on the above-mentioned "old-new" topics. We sincerely hope that it will provide readers with interesting new data as well as with comprehensive up-to-date reviews. We wish our readers pleasant and inspiring reading.

\section{Irena Rektorova Dag Aarsland K. Ray Chaudhuri Antonio P. Strafella}

\section{References}

[1] P. Barone, A. Antonini, C. Colosimo et al., "The PRIAMO study: a multicenter assessment of nonmotor symptoms and their impact on quality of life in Parkinson's disease," Movement Disorders, vol. 24, no. 11, pp. 1641-1649, 2009.

[2] K. R. Chaudhuri, C. Prieto-Jurcynska, Y. Naidu et al., "The nondeclaration of nonmotor symptoms of Parkinson's disease to health care professionals: an international study using the nonmotor symptoms questionnaire," Movement Disorders, vol. 25, no. 6, pp. 697-701, 2010.

[3] K. R. Chaudhuri and A. H. Schapira, "Non-motor symptoms of Parkinson's disease: dopaminergic pathophysiology and treatment," The Lancet Neurology, vol. 8, no. 5, pp. 464-474, 2009.

[4] H. Braak, K. del Tredici, U. Rüb, R. A. I. de Vos, E. N. H. Jansen Steur, and E. Braak, "Staging of brain pathology related to sporadic Parkinson's disease," Neurobiology of Aging, vol. 24, no. 2, pp. 197-211, 2003.

[5] G. Halliday, M. Hely, W. Reid, and J. Morris, "The progression of pathology in longitudinally followed patients with Parkinson's disease," Acta Neuropathologica, vol. 115, no. 4, pp. 409415, 2008.

[6] D. Aarsland, U. Ehrt, and I. Rektorova, "Cognitive and psychiatric disturbances in Parkinsons disease," Aging Health, vol. 7, no. 1, pp. 123-142, 2011.

[7] M. A. Hely, W. G. J. Reid, M. A. Adena, G. M. Halliday, and J. G. L. Morris, "The Sydney Multicenter Study of Parkinson's disease: the inevitability of dementia at 20 years," Movement Disorders, vol. 23, no. 6, pp. 837-844, 2008.

[8] A. L. Benabid, S. Chabardes, J. Mitrofanis, and P. Pollak, "Deep brain stimulation of the subthalamic nucleus for the treatment of Parkinson's disease," The Lancet Neurology, vol. 8, no. 1, pp. 67-81, 2009.

[9] A. J. Lees, J. Hardy, and T. Revesz, "Parkinson's disease," The Lancet, vol. 373, no. 9680, pp. 2055-2066, 2009. 


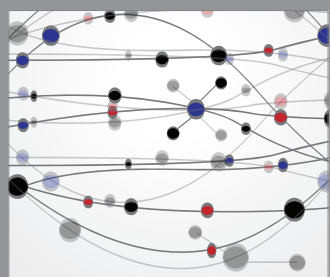

The Scientific World Journal
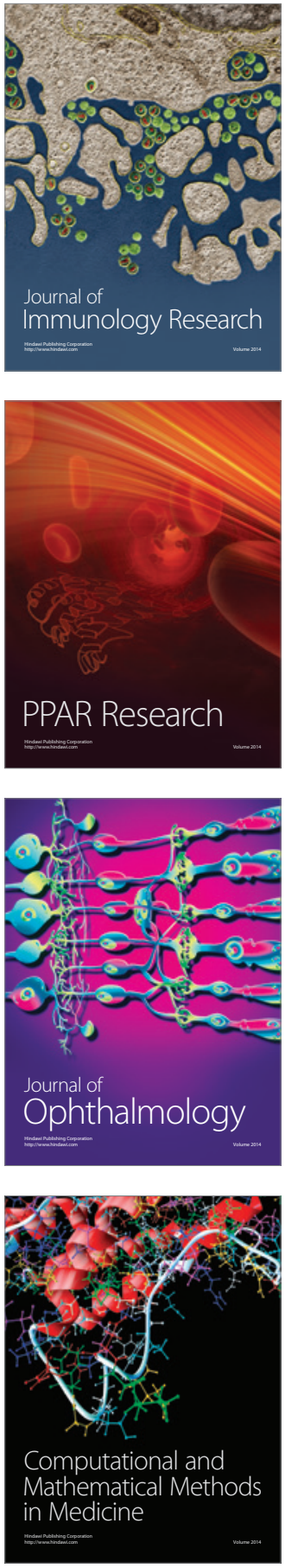

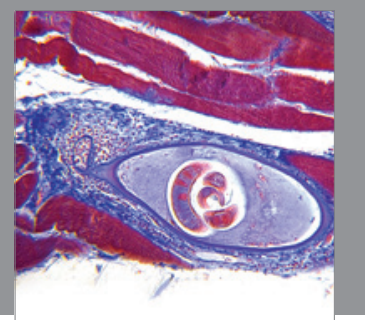

Gastroenterology

Research and Practice
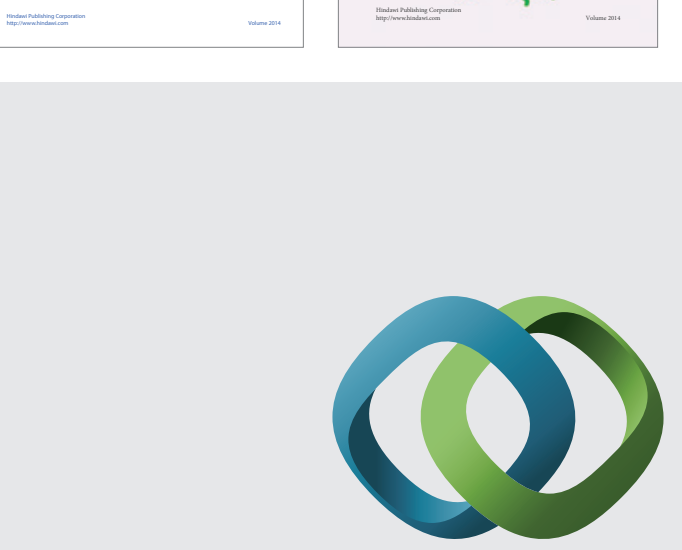

\section{Hindawi}

Submit your manuscripts at

http://www.hindawi.com
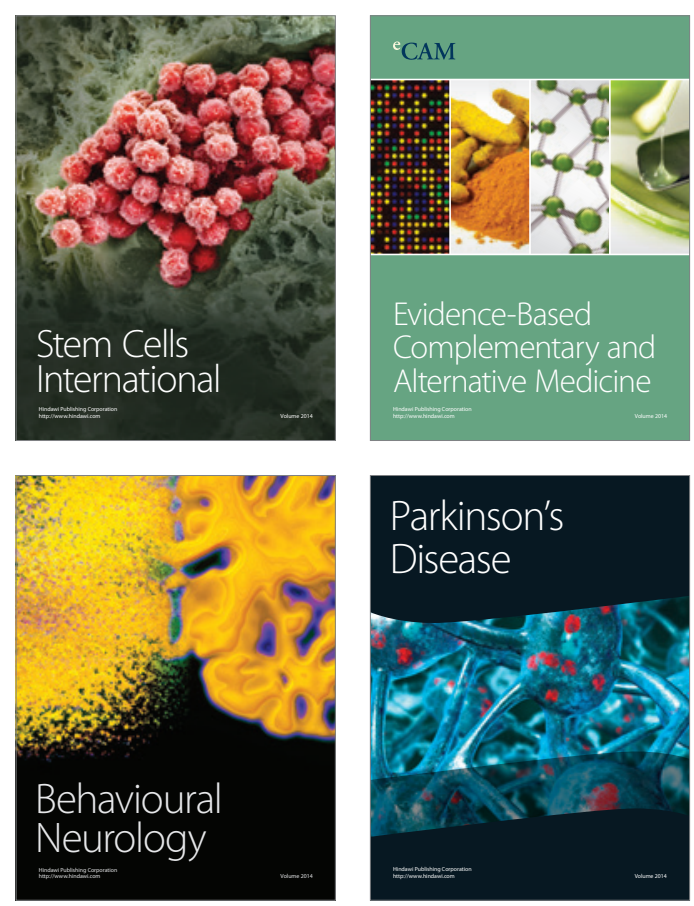

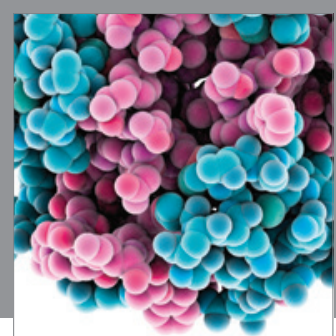

Journal of
Diabetes Research

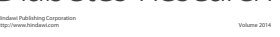

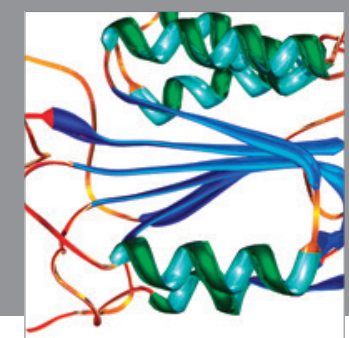

Disease Markers
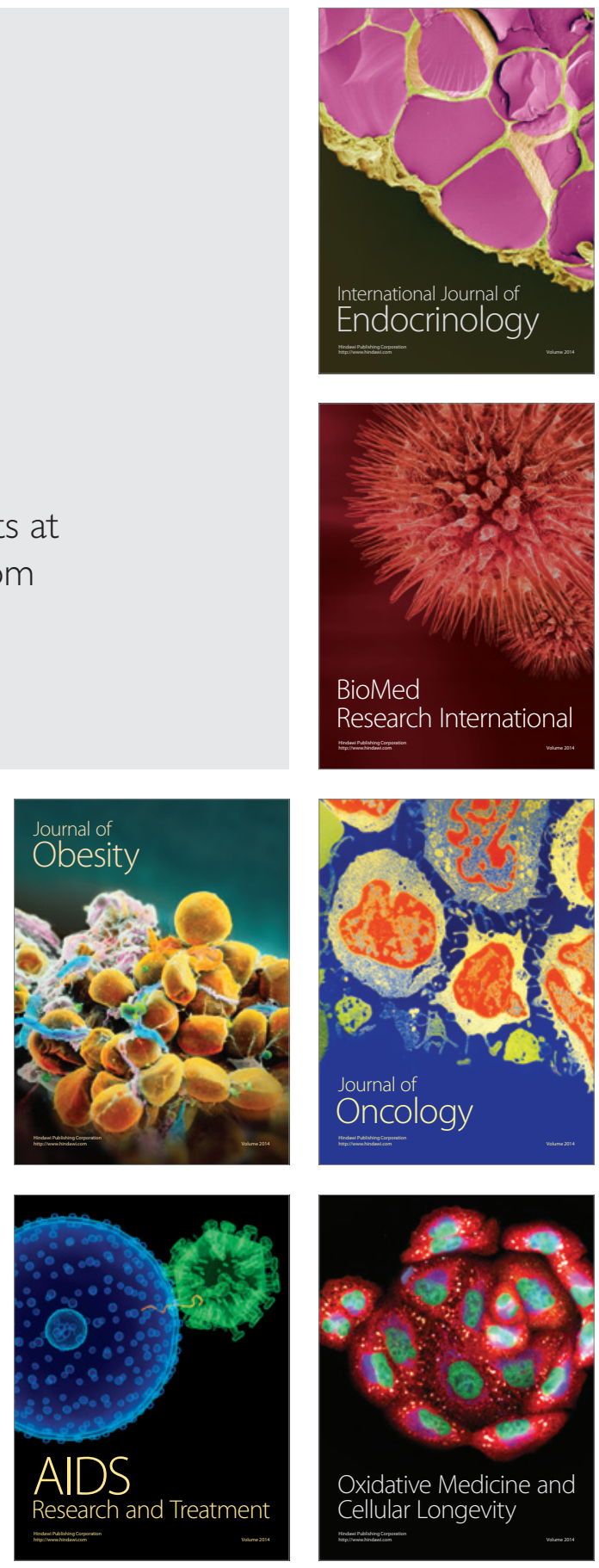